hier die Rheobase durch den in weiten Grenzen variierbaren anodischen Polarisationsstrom beliebig vorgegeben werden kann, was im System Eisen in konz. Salpetersäure nur durch Variation der Salpetersäurekonzentration in beschränktem Maße möglich ist.

$\mathrm{Da}$ für die Aktivierung des Eisens die Blairsche Gleichung ziemlich gut erfüllt ist, wird deutlich, wenn $t_{\mathrm{a}}$ in Abhängigkeit von $\log I^{-} /\left(I^{-}-I^{+}\right)$ aufgezeichnet wird (s. Abb.13). Es ergeben sich hierbei in guter Annäherung Geraden.

K. F. B o n h o ef fer und V. $\mathrm{H}$ a a s e $\mathrm{e}^{18}$ haben kürzlich nachgewiesen, daß die für den Nerven charakteristische Eigenschaft der Akkomodation (= Rheobasenerhöhung durch unterrheobasische Reize) auch am Ostwald-Lillieschen Nervenmodell vorhanden ist. Das System Eisen in Schwefel* säure ist im Unterschied dazu praktisch akkommodationsfrei, wovon man sich leicht überzeugen kann, wenn man sich vorstellt, daß unterrheobasische kathodische Ströme $\left(I^{-}<I^{+}\right)$hier nichts anderes bedeuten als eine zeitweilige Verringerung der anodischen Vorpolarisierung. Eine solche Anderung hat jedoch keinen merklichen Einfluß auf die Lage der Rheobase; denn bej weiterer Steigerung von $I^{-}$setzt unabhängig da. von, wie langsam diese geschieht, die Aktivierung ein, wenn $I^{-}=I^{+}$ist. Es lassen sich also keine stärkeren Dauerströme als $\mathrm{I}^{-}=I^{+}$, ,einschleichen", ohne daß Aktivierung erfolgt. Bei den hier in Frage kommenden anodischen Polarisations-

18 V. H a a se, Diss. Leipzig 1948; K. F. B o n ho ef fer, V. Ha a s u. G. L a $\mathrm{g}$ h a m m e r, Z. Elektrochem. angew. physik. Chem. 52, 29 [1948]. strömen $\left(0,1-300 \mathrm{~mA} / \mathrm{cm}^{2}\right)$ tritt nach erfolgter Aktivierung keine Repassivierung und damit auch keine Regenerierung zu erneuter Erregbarkeit (Aktivierbarkeit) des Systems ein. Aus diesem Grunde darf man unter den genannten Bedingungen auch kein periodisches Verhalten erwarten.

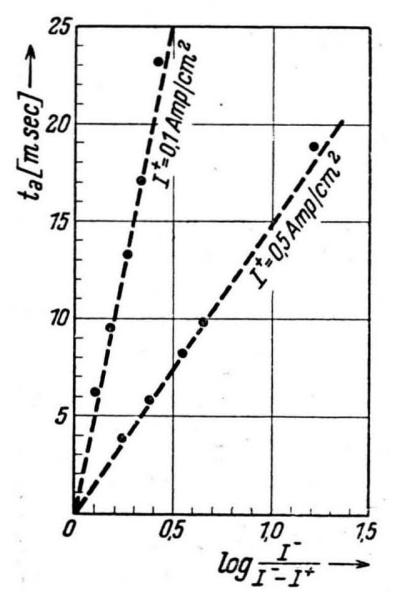

Abb. 13. Die Aktivierungszeit in Abhängigkeit von $\log \frac{I^{-}}{I^{-}-I^{\top}}$.

Dieses wird vielmehr erst bei höheren anodischen Stromdichten $\left(I^{+}>I_{0}\right)$ und besonders bei Gegenwart aktivierender Zusätze (wie z. B. Chlorionen) zum Elektrolyten möglich. Von diesen Umsiänden sollte jedoch bei den vorliegenden Untersuchungen zunächst noch abgesehen werden.

Hrn. Prof. K. F. B o n h o e f f e r danke ich für wertvolle Diskussionen und sein förderndes Interesse an dieser Arbeit.

\title{
NOTIZEN
}

\section{Zur Ausbeute-Steigerung bei der Kanalstrahl- Ionenquelle}

Von Det lef K a m ke

Physikalisches Institut der Universität Marburg

(Z. Naturforschg. 4 a, 391-293 [1949]; eingeg. am 25. Mai 1949)

Im Jahre 1938 hat Ha il e r ${ }^{\mathbf{1}}$ die Ausbeute der ganzmetallischen Kanalstrahl-Entladungsrohre durch Ein-

1 C. H a il e r, Wiss. Veröff. Siemens XVII, 3, 115 [1938].

2 Auch Henderson, Physic. Rev. 58, 192 [1940], berichtet über eine Ausbeutesteigerung durch Fokussierung der Ionen im Entadadungsraum, jedoch wird keine Beschreibung der Anordnung gegeben, auch fehlen Zahlenangaben über die Größe der Ausbeutesteigerung. bau einer Anodenblende erheblich verbessert ${ }^{2}$. Diese Anodenblende, die das kathodenseitige Ende der Anode abschlielst, hat einige $\mathrm{mm}$ Abstand von der Kathodenfläche (Abb. 1 a) und besitzt eine Mittelbohrung von etwa $10-15 \mathrm{~mm} \varnothing$ genau gegenüber dem Kanaleingang. Durch die Blende werden die Äquipotentialflächen vor der Kathode stark zusammengedrängt; sie verlaufen im übrigen so, daß sie eine Fokussierung der Ionen auf den Kanaleingang hervorrufen, wodurch der der Entladung entnehmbare Strom stark ansteigt. Allerdings steigt durch den Einbau der Anodenblende auch der zur Aufrechterhaltung der Entladung notwendige Gasdruck stark an und demzufolge die durch den Kanal strömende Gasmenge, die im Nachbeschleunigungsraum dann eine erhöhte Pumpleistung erfordert. Will man daher die 
Gasdurchströmung gering halten, so muß man beim Einbau der Anodenblende zu kleineren Kanaldurchmessern übergehen, wodurch die Ausbeute wieder herabgesetzt wird.

Es wurde daher untersucht, ob man durch passende Formgebung des Kanaleinganges eine Ausbeutesteigerung erzielen kann, die nicht durch starken Druckanstieg in der praktischen Verwertung behindert wird. Dabei wurde davon ausgegangen, daß man, um eine Fokussierung der Ionen $\mathrm{zu}$ erreichen, den Potentialflächen vor der Kathode eine starke Krümmung geben muß. Dies kann man außer durch eine Anodenblende auch erreichen, indem man dem Kanaleingang einen kleinen Kegel aufsetzt (Abb.1b). Die beste Kegelform gewinnt man dann, indem man bei festen Kanaldimensionen den aus der Entladung austretenden Ionenstrom in Abhängigkeit z. B. von der Höhe des Kegels' mißt (der $\varnothing$ der Kegelbasis wurde hier immer zu $8 \mathrm{~mm}$ gewählt, die Kegelhöhe bis zu $5 \mathrm{~mm}$ ).

Die Messungen wurden an einem Kanalstrahlrohr dés von $\mathrm{Lukanow}$ und $\mathrm{Sch} \ddot{\mathrm{utz}} \mathrm{e}^{3}$ verwendeten

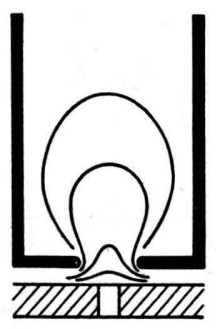

a

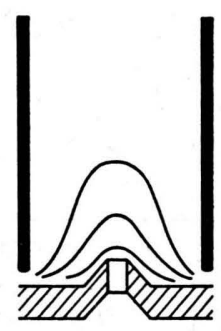

b
Abb. 1. Äquipotentialflächen vor der Kathode (a) mit Anodenblende, (b) mit Kanal-,Hütchen“.

Typs mit Wasserstoff als Entladungsgas vorgenommen (Anodendurchmesser $64 \mathrm{~mm}$, Anodenlänge $175 \mathrm{~mm}$ ). Die Anode konnte relativ zur Kathode mittels Stellschrauben so justiert werden, daß der Ionenstrahl wirklich auf den Kanaleingang gerichtet war. Die Messung der Entladungsspannung geschah vor einem Schutzwiderstand von $100 \mathrm{kOhm}$, so daß bei $10 \mathrm{~mA}$ Entladungsstrom die am Rohr liegende Spannung um $1 \mathrm{kV}$ niedriger ist als die gemessene (die unten angegebenen Spannungen geben die Werte von Rohr plus Vorwiderstand an). Die Entladung wurde über ein $\mathrm{Pd}$ Röhrchen mit Wasserstoff gespeist und vor jeder Messung 3 Stdn. eingebrannt. Der Ionenstrom wurde in einem Faraday-Becher etwa $20 \mathrm{~cm}$ hinter der Kathode gemessen. Zur Abschirmung der Sekundärelektronen wurde vor den Auffänger eine Blende $300 \mathrm{~V}$ negativ gegen diesen gebracht.

Für einen Kanal von $7 \mathrm{~mm}$ Länge und $1 \mathrm{~mm} \varnothing$ ist der gemessene Ionenstrom $i$ in Abhängigkeit von der Entladungsstromstärke $I_{\mathrm{E}}$ in Abb. 2 für die verschiedenen Kegelhöhen aufgezeichnet. Man sieht, daß eine Ausbeutesteigerung tatsächlich auftritt und daß sie

3 H. Luk anow u. W. S ch ü tz e, Z. Physik 82, 610 [1933].

${ }^{4}$ Die Konstanz des Kanaldurchmessers wurde mit dem Mikroskop überprüft.

\begin{tabular}{|c|c|c|c|c|c|c|}
\hline & $U_{\mathrm{E}}$ & $\begin{array}{c}1 \\
{[\mathrm{~mA}]} \\
\text { in } \%\end{array}$ & $\begin{array}{c}2 \\
{[\mathrm{~mA}]} \\
\text { in } \%\end{array}$ & $\begin{array}{c}4 \\
{[\mathrm{~mA}]} \\
\text { in } \%\end{array}$ & $\begin{array}{c}6 \\
{[\mathrm{~mA}]} \\
\text { in } \%\end{array}$ & $\begin{array}{c}8 \\
{[\mathrm{~mA}]} \\
\text { in } \%\end{array}$ \\
\hline $1,0 \mathrm{~mm} \varnothing$ & $20 \mathrm{kV}$ & 50 & 52,4 & 67 & 76 & 79 \\
$7 \mathrm{~mm}$ lang & $30 \mathrm{kV}$ & 33,8 & 34,5 & 44,5 & 50 & 53 \\
$1,0 \mathrm{~mm} \varnothing$ & $20 \mathrm{kV}$ & 49 & 51,5 & 49,4 & 60 & 68,8 \\
$4 \mathrm{~mm}$ lang & $30 \mathrm{kV}$ & 25 & 35,2 & 47,2 & 52 & 52,8 \\
\hline
\end{tabular}

Tab. 1. Prozentuale Ionenstromerhöhung.

einen kontinuierlichen Gang mit der Kegelhöhe zeigt ${ }^{4}$. In Tab. 1 sind die prozentualen Erhöhungen $\Delta i / i_{0}$ $\left(i_{0}=\right.$ Ionenstrom bei Kegelhöhe Null) für Kanäle von $1 \mathrm{~mm} \varnothing$ und 4 bzw. $7 \mathrm{~mm}$ Länge für die jeweils' beste

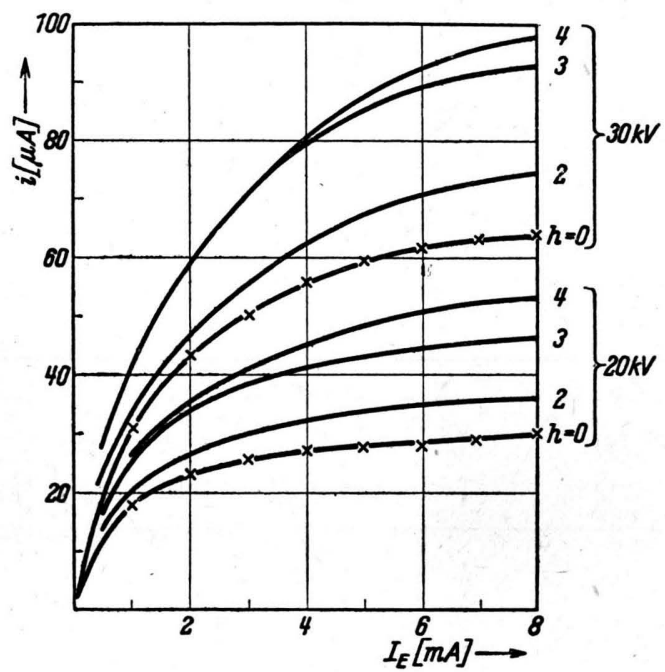

Abb. 2. Ionenstrom in Abhängigkeit vom Entladungsstrom. $h=$ Kegelhöhe in $\mathrm{mm} ; h=5$ und $h=4$ ergeben dieselben Werte.

Form dargestellt (Kegelhöhe $4 \mathrm{~mm}$ ). Auch bei dem dritten untersuchten Kanal $(1,5 \mathrm{~mm} \varnothing)$ wurde eine Steigerung des Ionenstromes gemessen, allerdings maximal nur um $37 \%$.

Im Zusammenhang mit den Hailerschen Untersuchungen ist das Verhalten des Gasdrucks interessant. Da zur Druckmessung lediglich ein McLeodsches Manometer zur Verfügung stand, wurden die Druckanstiege nur bei dem Wert von $4 \mathrm{~mA}, 30 \mathrm{kV}$ verfolgt. Bei den besten Kegelformen war die Druckerhöhung maximal $11 \%$. $\mathrm{Zu}$ erklären ist die gefundene Ausbeuteerhöhung mit einer durch die starke Potentialflächenkrümmung kurz vor der Kathode eintretenden Fokussierung, die wahrscheinlich die Bündelapertur herabsetzt. Daß die Ausbeuteerhöhung nicht dadurch zustande kommt, daß der Eingang des Kanals einfach $4 \mathrm{~mm}$ höher liegt, wurde dadurch kontrolliert, daß der ganze Kanalzapfen $(\varnothing 50 \mathrm{~mm})$ mit flachem Kanal um $4 \mathrm{~mm}$ höher gesetzt wurde. Dabei fiel die Ausbeute wieder auf $i_{\mathbf{0}}$. Außerdem ist nach den Messungen von 
$\mathrm{H} \mathrm{u}$ be r und $\mathrm{Met} \mathbf{z}$ g e ${ }^{\mathbf{5}}$ der schmalste Bündelquerschnitt der Ionen für $\mathrm{H}_{2}$ etwa $2 \mathrm{~mm}$ hinter dem Kanaleingang, so daß es sich hier nicht um ein besseres Heranschieben des Kanaleinganges an den „Brennpunkt" des Ionenbündels handeln kann.

Hrn. Prof. Walcher danke ich für wertvolle Anregungen und Diskussionen.

5 P. Huber u. F. Metzger, Helv. physica Acta 19, 200 [1946].

\section{Über komplementäre Beugungsprobleme}

\author{
Von A.-W. Ma u e*
}

(Z. Naturforschg. 4 a, 393-394 [1949]; eingeg. am 4. Juli 1949)

Nach dem Babinetschen Theorem lassen sich die Beugungserscheinungen an zwei ebenen komplementären, d.h. sich zur vollen Ebene ergänzenden Schirmen I und II aufeinander zurückführen. Dabei sind die auf I und II geltenden Randbedingungen als gleich, die einfallenden Wellen, die an den beiden Schirmen entsprechende Beugungsvorgänge hervorrufen, hingegen als verschieden angenommen ${ }^{1}$. Eine etwas einfachere Formulierung derselben Zusammenhänge ergibt sich bei Verwendung der gleichen einfallenden Welle, aber verschiedenen (,komplementären“) Randbedingungen. Sie gestattet es beispielsweise, eine Öffnung durch eine Scheibe $\mathrm{zu}$ ersetzen, ohne an der einfallenden Strahlung etwas zu ändern, was für die rechnerische Behandlung von Beugungsproblemen praktisch sein kann. Allerdings ist die Aussage, im Gegensatz zum eigentlichen Babinetschen Satz, nicht experimentell nachprüfbar, da sich die komplementäre Randbedingung nicht verwirklichen läßt.

Die skalaren und elektromagnetischen Wellen mögen den Gleichungen

$$
\Delta u+k^{2} u=0 \text { und } \operatorname{rot} \mathfrak{E}=i k \mathfrak{g}, \quad \operatorname{rot} \mathfrak{g}=-i k \mathfrak{E}
$$

genügen, die Schirme in der $x$ - $y$-Ebene mit der Normalenrichtung $\boldsymbol{z}$ liegen.

Die Randbedingungen für die beiden komplementären Wellen $u_{\mathrm{I}}$ und $u_{\mathrm{II}}$ seien

$$
\frac{\partial u_{\mathrm{i}}}{\partial z}=0 \quad \text { auf I, } \quad u_{\mathrm{II}}=0 \quad \text { auf II . }
$$

Zerlegung in einfallende Welle $u_{0}$ und Beugungswelle liefert

$$
u_{\mathrm{I}}=u_{0}+u_{\mathrm{I}}^{b}, \quad u_{\mathrm{II}}=u_{0}+u_{\mathrm{II}}^{h}
$$

$u_{\mathrm{I}}^{b}$ und $u_{\mathrm{II}}^{b}$ sind durch (1) und die Ausstrahlungsbedingung festgelegt. Aus Symmetriegründen ist $u_{1}^{b}$ ungerade, $u_{\text {II }}^{b}$ gerade bezüglich $z$ und deshalb $u_{\mathrm{I}}^{b}=0$ auf II und $\frac{\partial u_{\mathrm{II}}^{b}}{\partial z}=0$ auf I, weil $u_{\mathrm{I}}^{b}$ auf II, $u_{\mathrm{II}}^{b}$ auf I

* (17b) Weil a. Rh., Riedlistr. 41.

1 Für den Fall elektromagnetischer Wellen vgl. M e i $\mathrm{n}$ e r, Z. Naturforschg. 3a, 506 [1948]. nebst ihren ersten Ableitungen stetig sein müssen. Für die Gesamtwellen folgt hieraus

$$
\frac{\partial u_{\mathrm{II}}}{\partial z}=\frac{\partial u_{0}}{\partial z} \quad \text { auf I, } \quad u_{\mathrm{I}}=u_{0} \quad \text { auf II }
$$

und aus (1) und (3)

$\frac{\partial}{\partial z}\left(u_{\mathrm{I}} \pm u_{\mathrm{II}}\right)= \pm \frac{\partial u_{0}}{\partial z}$ auf I, $u_{\mathrm{I}} \pm u_{\mathrm{II}}=u_{0} \quad$ auf II

Den Bedingungen (4) genügende Lösungen der Wellengleichung für $u_{\mathrm{I}}+u_{\mathrm{II}}$ und $u_{\mathrm{I}}-u_{\mathrm{II}}$ lassen sich sofort angeben:

$$
\begin{gathered}
u_{\mathrm{I}}+u_{\mathrm{II}}=u_{0} \\
u_{\mathrm{I}}(x, y, z)-u_{\mathrm{II}}(x, y, z)=u_{0}(x, y,-z) .
\end{gathered}
$$

Wegen (2) muß aber $u_{\mathrm{I}}-u_{\mathrm{II}}$ neben (4) der Ausstrahlungsbedingung genügen und ist hierdurch erst eindeutig festgelegt, ebenso $u_{\mathrm{I}}+u_{\mathrm{II}}$ nach Subtraktion von $2 u_{0}$. Diese Bedingung wird durch (5a) nur auf der Schatten-, durch (5b) nur auf der Lichtseite erfüllt. $\pm u_{0}(x, y,-z)$ ist die von der vollen $x-y$ Ebene reflektierte Welle $u_{0}^{(\mathrm{I})}$, refl. bzw. $u_{0}^{(\mathrm{II})}$, refl, wenn dort die Randbedingung des Schirmes I bzw. II gilt. Man kann also zusammenfassen:

$$
\begin{aligned}
& \text { Schattenseite: } u_{\mathrm{I}}+u_{\mathrm{II}}=u_{0}, \\
& \text { Lichtseite: } u_{\mathrm{I}}-u_{\mathrm{II}}=u_{\mathrm{u}}^{(\mathrm{I})} \text {, refl. }=-u_{0}^{(\mathrm{II})} \text {, refl. }
\end{aligned}
$$

Im elektromagnetischen Falle gelte entsprechend

$$
\mathfrak{F}_{\mathrm{I}}^{\text {tang. }}=0 \quad \text { auf I, } \quad \mathfrak{S}_{\mathrm{II}}^{\text {tang. }}=0 \quad \text { auf II }
$$

und

$$
\begin{array}{ll}
\mathfrak{E}_{\mathrm{I}}=\mathfrak{E}_{0}+\mathfrak{E}_{\mathrm{I}}^{b}, & \mathfrak{E}_{\mathrm{II}}=\mathfrak{E}_{0}+\mathfrak{E}_{\mathrm{II}}^{b}, \\
\mathfrak{S}_{\mathrm{I}}=\mathfrak{S}_{0}+\mathfrak{S}_{\mathrm{I}}^{b}, & \mathfrak{S}_{\mathrm{II}}=\mathfrak{Y}_{0}+\mathfrak{S}_{\mathrm{II}}^{b} .
\end{array}
$$

Die Beugungswellen sind durch (7) und die Ausstrahlungsbedingung festgelegt. $\mathfrak{F}_{I}^{b}, \mathfrak{S}_{\mathrm{I}}^{\prime \prime}$ genügt überall, mit Ausnahme der Fläche I, den Maxwellschen Gleichungen. Auf I ist die Tangentialkomponente von $\mathfrak{F}_{\mathrm{I}}^{b}$ wegen (7) stetig, so daß nur die Tangentialkomponente von $\mathfrak{S}_{\mathrm{I}}^{b}$ springt, entsprechend einem Flächenstrom, durch den man sich die Beugungswelle erzeugt denken kann. Dieser elektrische Flächenstrom auf I erzeugt ein Magnetfeld, das die Fläche II normal durchsetzt, so daß $\mathfrak{S}_{\mathrm{I}}^{b}$, tang. $=0$ auf II. Entsprechendes gilt für $\mathfrak{F}_{\text {II }}^{b}$ auf I. Für das Gesamtfeld folgt

$$
\mathfrak{E}_{\mathrm{II}}^{\text {tang. }}=\mathfrak{E}_{0}^{\text {tang. }} \cdot \text { auf } \mathrm{I}, \quad \mathfrak{S}_{\mathrm{I}}^{\text {tang. }}=\mathfrak{S}_{0}^{\text {tang. }} \text { auf II, }
$$

und wegen (7) und (9) weiter

$$
\begin{array}{ll}
\left(\mathfrak{F}_{\mathrm{I}} \pm \mathfrak{E}_{\mathrm{II}}\right)^{\text {tang. }}= \pm \mathfrak{F}_{0}^{\text {tang. }} & \text { auf I } \\
\left(\mathfrak{I}_{\mathrm{I}} \pm \mathfrak{S}_{\mathrm{II}}\right)^{\text {tang. }}=\mathfrak{H}_{0}^{\text {tang. }} & \text { auf II }
\end{array}
$$

